\title{
Civilisations
}

Revue internationale d'anthropologie et de sciences

humaines

44 | 1997

Les peuples des forêts tropicales

\section{La problématique de l'espace indigène}

L'exemple du front pionnier au Brésil

\section{Pierre Grenand}

\section{(2) OpenEdition}

\section{Journals}

Édition électronique

URL : http://journals.openedition.org/civilisations/1620

DOI : 10.4000/civilisations. 1620

ISSN : 2032-0442

Éditeur

Institut de sociologie de l'Université Libre de Bruxelles

\section{Édition imprimée}

Date de publication : 1 janvier 1997

Pagination : 116-125

ISBN : 2-87263-122-4

ISSN : 0009-8140

\section{Référence électronique}

Pierre Grenand, «La problématique de l'espace indigène », Civilisations [En ligne], 44 | 1997, mis en ligne le 29 juin 2009, consulté le 03 mai 2019. URL : http://journals.openedition.org/civilisations/1620 ; DOI : 10.4000/civilisations. 1620 


\section{LA PROBLEMATTOUUE}

DE L'ESPPCE NDDGGẼNE:

L'exemple du front pionnier

au Brésil

\section{Pierre GheNAMO}




\section{INTRODUCTION}

Les malheurs des populations indigènes du Brésil ne sont ni une nouveauté, ni un phénomène marginal. Les phénomènes que nous observons aujourd'hui en Anazonie et les discours qui s'y rattachent s'inscrivent dans le temps long, Dès lors où les premiers Européens posent le pied sur le littoral atlantique, des comportements pérennes, les justifications qu'on leur donnent ainsi que les réactions critiques qu' ils engendrent, se mettent en place. Nous disposons aujourd'hui d'une bonne documentation d'archives sur l'histoire de l'occupation de l'espace brésilien et sur le sort des Amérindiens depuis les premiers temps de la Conquête jusqu'à nos jours [Hemming, 1978, 1987]. Il n'est donc pas question en quelques minutes de résumer cette histoire dont d'autres ont traité avec brio.

Mon propos sera surtout de montrer, en prenant comme exemple le cas du Brésil, l'un des pays concernés par notre rapport «La situation des Populations Indigènes des Forêts Denses Humides» demandé par les Communautés Européennes, comment l'Occident s'est enfermé, ou bien se nourrit, ou bien ne parvient pas à s'évader - cela dépend à quel point de vue l'on se place d'une politique destructrice à l'égard de populations minoritaires non-étatiques. Le thème de l'éternelle conquête d'un espace considéré comme vierge, aujourd'hui couramment désigné par le terme de frontière, nous semble être le point de cristallisation de tout le reste.

Afin de mieux faire ressortir la permanence des phénomènes, mais surtout afin de mieux faire comprendre les enjeux actuels, je procéderai par va-et-vient entre exemples du passé et exemples du présent et je tenterai une comparaison avec un autre grand exemple de conquête d'un espace occupé par les Amérindiens, celui des États-Unis d'Amérique.

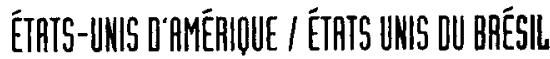

Malgré des occupations territoriales différentes, il s'agit dans les deux cas d'une même destruction culturelle et d'une volonté de conquête identique.

Même si un voyage vers l'Amérique du Nord nous éloigne quelque peu des forêts tropicales, la comparaison sera fructueuse, précisément parce que nous sommes face, d'un côté, au leader politique et économique du monde contemporain, de l'autre, à un pays en voie de développement, cependant l'un des plus dynamiques, et donc au cour des enjeux Nord/Sud... du moins si l'on s'en tient aux indicateurs économiques classiques. Le thème de la frontière occupe aujourd' hui une place importante, tant dans le discours officiel brésilien que dans le discours officiel nord-américain.

Dans le cas du Brésil, il s'agit d'un phénomène relativement récent, contrastant avec les formes anciennes d'occupation du territoire. Dans celui des F́tats-Unis, il s'agit de la nécessité vitale de maintenir en état de marche le vecteur idéologique de la construction d'une nation.

Dissemblables en apparence, ces deux frontières concernent pourtant l'une comme l'autre l'avenir de l'Amazonie, car si au départ les schémas d'occupation et les idéologies qui les soustendent sont bien différents, c'est néanmoins de cette différence que vont émerger certaines attitudes contemporaines essentielles. Un long détour par l'histoire s'impose. 


\section{Phase I: frontière nord-américaine eł géant brésilien}

La colonisation anglo-saxonne de l'Amérique du Nord, amorcée seulement vers 1640 , sera d'abord une lente puis de plus en plus rapide occupation agricole, fondée basiquement sur le colon et sa famille mettant en valeur un terroir. L'industrialisation suivra progressivement sur un territoire préalablement pleinement occupé. Bien sûr, l'espace sera ouvert par les coureurs de bois, les transporteurs de bétail, les chercheurs d'or, les aventuriers de tous poils ainsi que l'armée. Partout pourtant le poste, le fort, le village-champignon, feront toujours rapidement place à la communauté agricole organisée autour de ces quatre symboles étatiques que sont le juge, le pasteur, l'institutrice et le banquier. Les Amérindiens sont repoussés lorsque l'espace est encore vaste; ils sont combattus militairement lorsque l'espace s'essouffle; dans ce second cas, dès lors où l'on considère qu' ils ne présentent peu ou plus de danger, ceux qui restent sont circonscrits sur des territoires le plus souvent exsangues, les réserves. Dès la fin du XVIII siècle, il en existe sur la côte Est. Pour parler en termes d'économie, si les savoirfaire amérindiens sont exploités (guides pour les expéditions militaires, chasseurs de fourrure...), il n'est jamais sérieusement envisagé d'utiliser leur force de travail pour la mise en valeur des terroirs. Autrement dit, il n'est pas question de les intégrer dans le système colonial. Certes plus tard, au XIXe siècle, on pense que leur seule chance de survie est de les convertir en éleveurs et agriculteurs, mais c'est sans illusion, tout au plus pour les «guérir» de leur esprit nomade incompatible avec la cristallisation définitive des terroirs agricoles [Washburn, 1988].
Au Brésil, rien de tout cela. Tout d'abord il convient de rappeler, et le fait pèsera lourd dans la suite de mon propos, que ce pays fut pénétré dès 1500 par les Portugais, soit plus d'un siècle avant l'Amérique du Nord. De cette date à nos jours, le souci constant de la couronne portugaise puis de l'État brésilien sera d'agrandir et surtout d'assurer les limites de cet immense domaine, face aux possessions espagnoles puis face aux républiques qui en découleront. Bien qu'aujourd'hui encore, ce souci mobilise les énergies au détriment d'un développement prétendu méthodique, jusqu'au XIXe siècle, il accapara littéralement les forces vives de l'État.

Au Brésil, la colonisation ne fut jamais basée strictement sur l'agriculture : elle concerna tantôt des plantes cultivées (canne à sucre, café), tantôt aussi des produits miniers (or, diamant, fer...), tantôt enfin des produits de cueillette (cacao, caoutchouc...).

Surtout, la colonisation ne se fit pas en front; on y observa au contraire, et de façon répétitive, une volonté d'occupation en tâches, nommée hier cycles, aujourd'hui pôles, mais toujours fondée sur une volonté étatique et régulièrement sujvis d'échecs : c'est ainsi que l'industrie sucrière du Nordeste sera remplacée par l'exploitation des mines du Minas Gerais; celles-ci par la zone caféière de São Paulo. Aujourd hui on parle du Projet Gran Carajas, du Polonoroeste, etc, bien qu'ils soient partie intégrante d'une frontière économique et géographique.

En termes de société, on observa partout la même production d'une société violente et pauvre en quête d'espoir ou de richesses faciles et qui, à la différence des États-Unis, perdurera partout 
après la disparition des pôles de colonisation : c'est ainsi que le jagunço, «le tueur à gages», est une figure sociale emblématique plus unitaire du Brésil que le prêtre ou l'instituteur. Né il y a deux cents ans dans le Nordeste, il persiste dans sa région d'origine cependant qu'il fleurit aussi aux limites de l'espace brésilien en Acre ou au Roraima.

Quelle est la place faite aux Amérindiens dans la genèse d'un tel univers? Certes, si l'on se contente des thèmes classiques que sont l'extinction par maladie ou la violence subie, on notera une grande similitude du destin des populations autochtones des États-Unis et du Brésil. Et pourtant dans ce dernier pays, la place des Amérindiens est centrale. Tout simplement parce que, tant l'expansion territoriale de l'espace luso-brésilien que les tentatives de colonisations agricoles se sont d'abord faites avec leur force de travaiil, bientôt relayée sur la côte, mais sur la côte seulement, par celle des Noirs importés d'Afrique. Même si cette tendance s'amenuisa avec le temps, faute du maintien d'un peuplement indigène important, elle demeure réelle aujourd'hui encore, là où les Amérindiens sont encore présents de manière significative [Ribeiro, 1986].

On peut mettre en parallèle avec cette appropriation de la force de travail, la négation ou la remise en question des espaces indigènes pendant toute la période qui va du XVIe au milieu du XIX" siècle. En effet, même si l'on confie très souvent aux religieux la pacification des populations indigènes (il est tout de même préférable de disposer d'une force de travail intacte), on remet inéluctablement en question leur tendance à créer des espaces autonomes où ils vivent seuls avec les
Amérindiens. Ie cas extrême le plus illustrant est bien sûr celui des Jésuites qui, ayant réussi à transformer les Indiens Guarani en producteurs efficaces selon les normes européennes, furent expulsés et leurs ouailles réprimées par une force armée hispano-portugaise. Leur faute avait été de transformer les Guarani en une unité de production autosuffisante et d'avoir créé une structure politique autonome. Cet épisode mérite pour le moins d'être médité à l'heure du développement alternatif!

\section{Phase 2 : quand le Brësil découvre la frontière} "àlaméricaine".

Du point de vue sociologique, le Brésil est un univers fascinant : il s'agit d'un cas, peut-être unique au monde, de «société-sandwich». Plus qu'une mosaïque culturelle, une société de tous les métissages si bien chantée par les poètes et écrivains du monde occidental, on peut observer dans le Brésil contemporain des micro-situations qui reproduisent chacune des phases de son histoire, comme si les événements du passé ne pouvaient servir de leçon; ou plutôt comme si ce qui est arrivé là-bas avait toute raison de se reproduire ici. Là est le lieu de la mosaïque brésilienne.

Dès les débuts de son Indépendance, le Brésil comme la quasi-totalité des États d'Amérique latine, dut constater rapidement sa dépendance économique voire militaire envers l'Europe nonihérique et les États-Unis. Pis encore, c'était bien au monde occidental qu'il accrochait son rêve de construction étatique, puisqui on lui offrait un idéal en kit, l'idéal républicain, la seule alternative avec ce qu'il prétendait rejeter, si l'on excepte quelques remous nativistes. Notons que cet idéal, 
via des subtilités gréco-romaines qui le qualifient de démocratique, est devenu aujourd'hui dans le langage de M. Bush revu et corrigé par M. Clinton, le seul idéal planétaire concevable. Cet idéal en kit apportait dans ses malles les notions de Droits de l'Homme, d'égalité devant la loi, de respect des devoirs civiques. Notions fondamentales, certes, personne ne se permettrait d'en disconvenir, notions subversives même, et pourtant, notions ambiguës, équivoques, et, on va le voir, presque suspectes.

Pourtant, si le Brésil indépendant conserva son schéma d'occupation spatiale et surtout ses structures sociales antérieures, se contentant d'y plaquer l'idéal républicain, des changements de fond allaient progressivement émerger : en particulier au moment même où la notion de protection des Amérindiens apparaît dans le discours officiel, surgit également, 150 ans après les ÉtatsUnis d'Amérique, la notion de frontière, traduite ici en tant que doctrine de l'occupation du territoire national. Les Luso-brésiliens étaient présents, on s'en souvient, depuis 1500; ce n'est qu'à la fin du XIXe siècle qu'ils s'avisent d'occuper, de remplir, leur territoire national : or, il ne s'agit pas de faire rouler les chariots, d'avancer dans l'inconnu en laissant derrière soi des jalons utilisables par d'autres; l'image qui convient le mieux à mon sens est la suivante : le Brésil décide d'écarter les bras, de toucher toutes ses frontières en même temps, car le territoire est déjà maillé. Une histoire chaotique y a déjà roulé ses flots troubles; les Amérindiens, même s'ils voient leur nombre s'amenuiser sans cesse, sont toujours là, désormais éparpillés, côtoyant bon gré mal gré des millions de métis. Peuplant l'Amazonie brésilienne, les Caboclo sont le résultat de toutes les avanies dont soient capables les hommes, ${ }^{[1]}$ mais certainement pas le produit d'un compromis, voire d'une alliance entre l'homme blanc et la femme amérindienne, comme le rêveront pius tard les poètes et les écrivains brésiliens, suivis sans discernement par un lot de penseurs uccidentaux [Grenand \& Grenand, 1990].

Dés lors, pourquoi s'étonner de l'absence d'une société construite dans l'intérieur du Brésil? Euclides da Cunha l'avait déjà compris à la fin du XIXe siècle dans son superbe livre « 0 s Sertões" [1993]; Blaise Cendrars, qui avait connu cet univers, reprit l'idée vers 1920. La réponse était simple : l'immensité de ce pays n' avait pas participé aux évolutions successives de l'Étal. Autrement dit, il n'y avait pas de patrie. N' avons-nous pas entendu en 1987 encore cette interrogation simple proférée à $15 \mathrm{~km}$ seulement de Manaus, ville d'un million d'habitants et capitale de l'État d'Amazonas, par une femme, maraîchère dynamique. de surcroît influente politiquement : «Doit-on traverser la mer pour se rendre à Rio de Janeiro?"

Il est essentiel de considêrer que l'idéal brésilien de colonisation de l'espace accompagne celui de domestication de l'Amérindien. Vers les années 1880, alors que l'on passe sans heurts de l'Empire à la République, des forfaits absolument sans appel ont été commis dans les trente années précédentes à l'égard des indigènes dans les vallées du Rio Negro, du Jurua et du Purus. C'est dans ce contexte bouillonnant qu'émerge une des plus attachantes parmi les figures emblématiques du Brésil : le républicain Rondon, général puis maréchal, mais aussi fils de femme amérindienne. 
Alors qu'il jalonne de poteaux télégraphiques des étendues stériles de l'immensité brésilienne, il s'avise d'inventer un concept génial, génial parce que parfaitement utopique, utopique parce qu'il est à la fois cohérent avec la construction de la société brésilienne et impossible à mettre en pratique : l'intégration progressive des Amérindiens à la société nationale. Dans cette optique, la reconnaissance d'une terre indigène n'est et ne peut être autre chose qu'une étape transitoire, sorte de salle d'attente vers la Civilisation. Et, depuis l'énonciation de ce concept, le Brésil ne cesse de vouloir intégrer, pathétiquement ou cyniquement, c'est selon les gouvernements, des hommes et des femmes qui ne veulent pas être intégrés.

Intégrés à quoi? $\lambda$ la société nationale. Quelle belle notion que celle-là, et pourtant, quel petit manteau de cache-misère! La réalité toute simple est aujourd'hui perceptible par chaque Amérindien. Il y a quelques années, j'eus le triste privilège, en Amapá, de franchir en camion quelques centaines de kilomètres d'une bretelle de la Iransamazonienne, la Perimetral Norte; je parcourus ainsi de bout en bout le front pionnier: derrière la ligne de défrichement marquée par des taches de mauvais brûlis d'où émergeaient des troncs calcinés, de misérables masures lapées sur le rebord de la piste abritaient des loques humaines, revêtues d'une grisaille rapiécée, certaines même flottant dans des sacs de jute. Puis, je me heurtai à un panneau annonçant la réserve indigène où je me rendais. La République Fédérale du Brésil y menaçait des foudres de l'enfer' quiconque y pénétrerait sans autorisation, quiconque y chasserait, y pêcherait, y chercherait de l'or, y débarderait du bois. Et la forêt, la grande forêt commençait ici; comme si c'était ce panneau qui, d'un seul coup, lui redonnait la vie. Quelques heures plus tard, j'arrivais dans un village amérindien : un havre de paix dans un océan de verdure; une société d'hommes et de femmes dans la plénitude de leur être; une langue, une culture, une philosophie de la vie qui affichaient clairement leur volonté de perdurer dans cet état.

Face à de telles images, que signific exactement l'invitation faite aux Amérindiens à intégrer la société nationale? Quelle nation indigène digne de ce nom pourrait accepter un tel marché où l'on essaie de lui vendre comme progrès minimal un modèle de société composée basiquement de misérables? Nous sommes ainsi inexorablement revenus au XVle siècle, où l'offre proposée aux Amérindiens était déjà la même.

\section{DE l'NDIEN EN RÉGERVE À l'INDIEN PROBUCTIVISTE}

La frontière économico-géographique et la frontière idéologique se rejoignent en un seul chœeur pour chanter à l'unisson. Dés l'époque de sa création, la notion de territoire indigène put être interprétée de deux manières : pour ses promoteurs, c'est la version humaniste, la réserve était un lieu où l'Amérindien devait se civiliser volontairement et progressivement. Pour ceux directement intéressés par l'exploitation de l'espace amazonien, c'est la version capitaliste, elle était l'endroit où ils étaient rassemblés après leur pacification, afin de laisser libre l'accès du territoire environnant. À cette notion de réserve, fut dès le départ attachée celle de poste indigène qui reste, aujourd'hui comme hier, un lieu d'attraction : c'est là que l'Amérindien, attiré comme une 
alouette par son miroir, se familiarise avec les pratiques et les objets du monde extérieur; c'est surtout le lieu où il se sédentarise. Bien souvent, les concentrations que le poste indigène engendre laissent déserte une partie non négligeable de la réserve. D'où la possibilité de rapidement affirmer (les citations abondent) que les Amérindiens disposent d'un bien trop grand territoire.

Les Amérindiens sont alors invités à se transformer en producteurs extractivistes, au mieux all service de l'État, au pire pour le bénéfice d'un patron auquel on les loue. Ils sont invités à rechercher caoutchouc, balata, peaux de jaguar, noix de Pará, plantes médicinales. Ainsi, le Brésil ne fait que perdurer dans l'ornière de ses débuts: on ne cherche absolument pas, malgré les textes, à transformer l'Amérindien en un citoven brésilien ordinaire mais à l'utiliser comme prolétaire après l'avoir appauvri et domestiqué. Or, dans le même mouvement, on n'assiste jamais à la réelle domestication de l'espace.

Lorsque l'Amérindien refuse de se soumettre. il devient le rebelle et bon à éliminer. Cependant, contrairement aux États-Unis, le Brésil républicain n’intervient pas. Il n'y a pas de guerres indiennes; l'État, officiellement s'entend, se dérobe et les forfaits perpétrés par l'armée sont cachés à l'opinion publique. La place est laissée aux propriétaires terriens et aux politiciens locaux. Cette inversion est significative : elle laisse apparaître une violence privée, contre laquelle le gouvemement peut, officiellement toujours, s'ériger en agitant de grands principes humanitaires.

Autrement dit, les dés sont pipés : d'un côté. le Brésil proclame la nécessité de couvrir l'espace amazonien sous le drapeau de l'Union; de l'autre,
l'État, sans cesse au bord de l'explosion sociale dans ses mégalopoles de la côte et dans le Nordeste, a besoin pour lui servir de soupape, de conserver un espace non civilisé, un espace où ne règnent ni loi ni justice, un espace oì les coups de force de tout ordre soient permis. Dans ce contexte, la frontière n'est pas géographique; elle est fantasmatique. Chacun court y retrouver ses rêves et nul développernent, au sens occidental du terme, n'en ressort.

La propre idée du maréchal Rondon, celle de terre indigène pensée comme pallier civilisateur, contenait en germe sa propre contestation, que l'on voit courir en filigrane avant qu'elle ne s'extériorise dans les années cinquante. Une fois encore, on rejoint ici l'Amérique du Nord. Au fur et à mesure que l'espace se rétrécit, que les coups portés aux Amérindiens se multiplient, la notion de terre indigène devient porteuse du concept d'inaliénabilité. Les terres laissées aux Amérindiens sont désormais appréhendées par ces derniers comme des pôles de la vie traditionnelle, des lieux de repli, des peaux de chagrin que l'on doit non seulement sauvegarder, mais sur lesquelles on doit au contraire s'appuyer, desquelles on doit partir pour tenter de les agrandir. C'est ainsi que va s'élaborer un discours brésilien complexe, bâti d'un côté sur les idées progressistes de démocratisation et d'égalité sociale, de l'autre sur l'idée frileuse d'indépendance nationale [Anonyme, 1983].

Toutes ces composantes vont exploser au temps de la dictature militaire. En même temps qu il étouffe et assassine les libertés individuelles, l'État va reprendre en l'amplifiant la nécessité pour les individus d'occuper définitivement 
l'espace amazonien. Nonobstant le constat scientifique selon lequel l'Amazonie n'est pas un biome capable de supporter une agriculture intensive, la dictature militaire sera la première à en organiser une tentative d'occupation. Ft de cette manière, elle va chercher à appliquer dans la réalité le concept de frontière dans son sens nordaméricain. En corollaire, elle va éteindre le SPI, Service de Protection des Indiens créé par le Maréchal Rondon, et mettre en place une nouvelle législation de lindien ainsi que l'organisme capable de la faire appliquer, la FUNAI, où derrière une apparente protection renforcée, va être mise en place une véritable politique de liquidation des territoires indigènes [Albert, 1992].

On n'en est pas sorti. Alors que la dictature s'est éteinte, qu'elle est désomais inscrite dans les pages des livres de l'Histoire du Brésil, les mêmes enjeux et les mêmes acteurs, quoique singulièrement brouillés par un discours moderniste, demeurent. Quelques thèmes d'actualité tels que libéralisme économique, développement durable ou protection de l'environnement, ne font que couvrir de leurs oripeaux des actes aussi vieux que le Brésil colonial et menacer les sociétés amérindiennes dans leur essence même.

Cette situation contemporaine est à la fois très bien documentée, ne serait-ce que par le travail de nombreuses $O N G$, mais elle est aussi confuse en raison principalement des dérives médiatiques qu'elle génère. J'en donnerai deux exemples parmi les plus significatifs, se rejoignant pleinement quant à leurs implications concrètes : la nouvelle Constitution démocratique brésilienne promulguée en 1989 prévoyait que la totalité des terres amérindiennes devait être déli- mitée en octobre 1993. Non seulement nous en sommes loin, mais entre-temps, nous avons pu assister dans le cas du territoire yanomami, sans oublier les violences pratiquées sur le terrain, à toutes les tentatives juridiques les plus retorses pour priver une population indigène de plus de $50 \%$ de son territoire. Ailleurs, les terres indigènes sont identifiées ou interdites, en attendant des jours meilleurs où l'occupation de facto par des migrants ou des chercheurs d'or permettra de réduire à néant cette protection de papier. Mieux encore, ce sont les propres militaires de l'École de Guerre qui promulguent une nouvelle doctrine, la «décennie démocratique», proclamant cette fois que les Amérindiens sont des «kystes anthropologiques» dont le but est de favoriser la mainmise des États du Nord sur l'espace amazonien [Albert, op. cit.]. Brouillage simpliste, perversion des mots, lorsque l'on sait que ce sont les mêmes forces politiques qui réclament aux banques du Nord les crédits pour la construction des grands barrages ou l'ouverture de nouvelles routes transcontinentales. Tout cela, rappelons-le, sur fond de frontière fantasmatique et surtout de croissance des métropoles urbaines où finissent par échouer tous les rêves amazoniens.

Rappelons cependant quelques chiffres, toujours pour le Brésil :

- $\quad 10 \%$ de la forêt amazonienne, selon les sources scientifiques les plus sûres, ont été à ce jour déforestés [Brown \& Brown, 1992];

- $\quad 16 \%$ de la forêt amazonienne sont revendiqués par les populations indigènes [Anonyme, 1991];

- $\quad 74 \%$ de la superficie de l'Amazonie forestière ne relèvent donc pas de la problématique indi- 
gène. Il en ressort que les Amérindiens ne sauraient constituer un quelconque obstacle au soidisant développement de l'Amazonie. L'Amérindien ne serait-il pas plutôt un modèle subversif pour la nation brésilienne?

Il ne s'agit pas là d'une provocation mais plutôt d'un recentrage du débat : depuis cinq ans, les mêmes forces politico-militaires brésiliennes qui vitupèrent contre les pays du Nord ont officiellement épousé le discours écologique, comme par exemple à travers le programme Nossa Natureza. Ne pas leur accorder de nouveaux prêts les empêcherait, selon elles, de mettre en place une véritable politique de protection de l'environnement; elles stigmatisent au passage l'agriculture sur brûlis des Amérindiens et des Caboclo, soidisant destructrice. De plus, assimilant parfatitement le discours scientifique, elles ont compris que le développement agricole était impossible et qu'il était infiniment plus intéressant de se concentrer sur le développement minier, facile à circonscrire géographiquement. Celui-ci présente l'avantage de libérer des espaces considérables et de drainer vers l'Amazonie des fonds venant du Nord autour de thèmes à la mode, tels que ressources alternatives, banques de gènes, plantes médicinales ou éco-tourisme. En prime, ce type de développement n'empêche pas de maintenir, dans l'esprit d'une population urbaine bouillonnante, le vieux mythe de l'Eldorado.

l.eur discours n'est pas dépourvu d'écho. Dans la mouvance du discours scientifique et des manifestations internationales de défense des minorités indigènes, est né précisément dans les pays du Nord, un intérêt tout nouveau pour les forêts tropicales et singulièrement celles du bassin amazonien. Sous prétexte de protection de la forêt, de conservation de la bio-diversité et de chances données aux populations indigènes de participer d'un développement soutenable, on a vu surgir ces dernières années des entreprises capitalistes aux appellations mirobolantes telles la Shaman Incorporation la Body Shop Incorporation,et la Cultural Survival Enterprise! D'autres, sous des noms plus neutres, telle la Fondation Yves Rocher, n'en poursuivent pas moins des buts identiques. Leur théorie est simple : s'ils n'entrent pas dans une économie de profit, les Amérindiens ne peuvent envisager de maintenir une économie soutenable. Un rapport récent de Corry [1993] a magistralement montré quels mécanismes de dépendance, rappelant ceux du passé, étaient sous-jacents dans ces projets et totalement contraires à l'autodétermination des populations des forêts tropicales.

\section{CONCLUSION}

On aura compris que depuis cing siècles, rien n'a profondément changé pour les Amérindiens du Brésil, sinon qu'aujourd hui, non seulement des forces internationales mais encore les propres organisations indigènes, prennent conscience que leur destin est similaire à celui de bien d'autres communautés sur terre. Une comparaison entre des Indiens d'Amazonie armés d'arcs et de flèches et des paysans européens manifestant contre le GATT ne serait pas aussi abusive qu'elle en a l'air. L'économie des Amérindiens d'Amazonie est perdurable (sustainable). Elle nous indique un choix de société qui remet en question la croissance et le productivisme à outrance; loin d'être un cas marginal, 
leur destin est profondément lié, que nous le voulions ou non, à nos choix de société contemporains.

Tant qu'un système de production est ontimal, et $j$ 'entends par optimal qu'il assure le bonheur existentiel de l'ensemble de ceux qui le pratiquent, il est criminel de vouloir le remplacer par un autre, surtout si ce dernier n'a jamais fait les preuves que de ses échecs.
NOTE

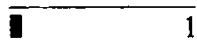

Remarque des éditeurs:

Seule la responsabilité des auteurs est engagée dans cette publication. En ce qui conceme le dêtat sur les caboclo, voir de IAVELEYE:

[ce volume] pour un autre édairage. 